\title{
Analysis of Access Delay and Delay Jitter for the CSMA/CA Mechanism with Inactive Period
}

\author{
Xuefen CHI, Minghuan ZHANG, Linlin ZHAO, Lei QIAN \\ Dept. of Communications Engineering, Jilin University, Nanhu Road 5372, 130012, Changchun, China \\ chixf@jlu.edu.cn, \{zhangmh16, zhaoll13, qianlei16\}@mails.jlu.edu.cn \\ Submitted July 1, 2018 / Accepted December 5, 2018
}

\begin{abstract}
Owing to the distributed features and flexibility, carrier sense multiple access with collision avoidance (CSMA/CA) mechanisms have been widely adopted in wireless networks. Considering energy consumption, we devote to exploring an energy efficient carrier sense multiple access with collision avoidance (EE-CSMA/CA) mechanism. The access delay and delay jitter are two of most important quality of service (QoS) metrics. EE-CSMA/CA inevitably experiences the tortures bred from its delay and delay jitter performance, because its frame structure with inactive period brings delay. In this paper, we aim to analyze the access delay and delay jitter of packets for the CSMA/CA with inactive period under the unsaturated conditions. Based on the Markov theory, we model the mechanism of EE-CSMA/CA as a four- dimensional Markov chain, and the fourth variable of Markov chain differentiates the active period and the inactive period of EECSMA/CA. Then, deriving in probability generation domain, we get the probability distribution of service time and obtain the access delay. Finally, the queue in a node is decoupled, and two queues with different states of empty and non-empty are configured logically, which helps to enable the complex analysis of delay jitter. Based on the whole probability analysis, the probability distribution of delay jitter is obtained. We show that the active ratio (active time over whole frame time) affects the access delay and delay jitter. When the active ratio is constant, the duration of active period has less effect on the access delay while it deteriorates delay jitter performance.
\end{abstract}

\section{Keywords}

CSMA/CA mechanism, inactive period, unsaturated conditions, Markov chain, access delay, delay jitter

\section{Introduction}

The fifth generation (5G) wireless networks are expected to support various services such as Voice over Internet Protocol (VoIP), real-time video streams [1], interactive online games and so on. Different applications have different quality of service (QoS) requirements. Real-time applications have requirements for delay and jitter of delay, for example, VoIP and Skype. The authors of [2] analyzed the delay of VoIP networks, and the authors of [3] stated that the Skype required to satisfy the QoS requirements in terms of not only delay but also delay jitter. It was reported that suffering from jittered voice, a lot of users hung up their calls due to the unintelligible voice conversation [3]. The analysis of the delay and the delay jitter is the essential preliminary step for the resource allocation of networks. Owing to the distributed features and flexibility, carrier sense multiple access with collision avoidance (CSMA/CA) mechanisms have been widely adopted in wireless networks. For the sake of energy efficiency, some of CSMA/CA mechanisms introduce the inactive period in the frame structure (such as protocols in IEEE 802.15 series). Nodes sleep and don't transmit packets during the inactive period. The introduction of inactive period can also improve the spectral efficiency of coexisting networks [4]. However, inactive periods inevitably incur the delay and delay jitter. The first step to analyze the delay and delay jitter of CSMA/CA mechanism is to model the service process. Due to the stochastic nature of the CSMA/CA mechanism, it is difficult to analyze the service process and the service time (access delay) of a packet. The delay jitter is involved with delay. The delay of every packet is random due to the collision, which complicates the analysis of delay jitter. We consider the CSMA/CA mechanism with inactive period, which increases another difficulty to the analysis of delay and delay jitter.

Several works adopted the queuing theory to analyze the average delay of packets. In these works, the service time was assumed to be a statistical process, such as the Markov process [5], independent and identical distributed (IID) process following exponential distribution [6] or general distribution [7]. Some works took the characteristics of the specific service mechanisms of networks into consideration. Inspired by Bianchi's seminal Markov model of CSMA/CA mechanism [8], the authors of [9] and [10] dealt with the average access delay analysis of the CSMA/CA mechanisms in saturation traffic scenarios. Considering the unsaturated traffic, the authors of [11] put forward an extended Markov model which added a state to abstract unsaturated situations, and analyzed the average access delay performance. The authors of [12] analyzed the delay of user datagram protocol (UDP) traffic jointly con- 
sidering the transmission control protocol (TCP) congestion control mechanisms. In recent years, energy issues have raised concerns. Some experts begin to explore the protocols with inactive period. The authors of [13] evaluated the average access delay performance of single-hop IEEE 802.15.4 networks.

Jitter of delay is another important QoS metric for real-time services, which is defined as the difference between the values of delay of two successive packets [14]. In the last decade, some studies have been devoted to estimating of delay jitter by the steady-state analysis method. Due to the complexity of the practical service mechanism, most of these works have been done in the context that the service time was abstracted as a certain distribution, for example, exponential distribution [15], [16] and deterministic distribution [17], [18]. Our team analyzed the average delay jitter for the tandem queuing system model, where the service time was assumed to be geometrically distributed [19]. However, the average delay jitter is one of features of the delay jitter, which cannot depict the delay jitter vividly. Hence, a transient analysis method for the delay jitter of packets was proposed in [20], where the service time was modeled as an IID process following exponential distribution. In our recent work [21], delay jitter of packets was estimated by transient analysis method in wired internet protocol (IP) concatenated networks, where a packet is served through exactly one slot in IP networks. In all aforementioned works, delay jitter issues of practical networks are not tackled.

In this paper, we propose an energy efficient CSMA with collision avoidance (EE-CSMA/CA) mechanism. In the EE-CSMA/CA mechanism, nodes don't sense the channel state during backoff periods and stay in sleep mode during the inactive period. The service time is the time to serve a packet. The challenge of the service time analysis for CSMA/CA lies in the space-relevance among the service processes of nodes. For the frame with inactive period, how to quantify the effect of frame structure on the service time of EE-CSMA/CA is still an unexploited problem. We define active ratio $R$ as the ratio of the duration of active period to whole frame duration, and investigate the effect of $R$ on access delay as well as delay jitter. On the other hand, the traffic arrival is unsaturated generally. For the EE-CSMA/CA, only the nodes having packets in queues take part in the contention, which changes the relevance of service processes of nodes. Hence, the unsaturated traffic situation makes the space-relevance of service processes of nodes more complicated. The service time of packets is random due to the collisions of packets. The randomness of the arrival and service makes the delay of every packet different. The delay jitter is involved with delay. The random feature of delay makes the analysis of delay jitter intractable. For handling the above issues, we devote to the analysis of the access delay and delay jitter of EE$\mathrm{CSMA} / \mathrm{CA}$ in this paper. Our main contributions are summarized as follows.
- For EE-CSMA/CA with inactive period, we model the service process of a packet as a four-dimensional Markov chain, and the fourth variable of Markov chain differentiates the period of the service process being active or inactive. Considering the unsaturated traffic situation, we model the number of packets in the queue of a node as a discrete time Markov chain, and obtain the probability of the queue being empty which affects the idle probability of the channel and the probability of successful transmission. Based on these derived probabilities, we obtain the probability distribution of the service time in probability generation domain.

- Depending on the probability distribution of the service time, we analyze the delay jitter of a packet. In unsaturated traffic conditions, the queue in a node can be decoupled into two states of empty and non-empty, which helps to enable the complex analysis of delay jitter. When the queue of node is empty, the delay jitter of a packet is associated with the service. When the queue of node is non-empty, the delay jitter is decided by the relation between the inter-arrival time of two sequential packets and the service time of the packet. Finally, we obtain the probability distribution of delay jitter relying on the whole probability analysis.

- We study the influence of frame configuration on the access delay and the delay jitter of packets. When the active ratio is constant, the duration of active period has less effect on the access delay while it deteriorates delay jitter. For energy saving and QoS guarantee, it is necessary to set the frame structure reasonably. Under the access delay and delay jitter constraints, we estimate suitable durations of active period (i.e. the maximum durations of inactive period) by the dichotomy, respectively.

This paper is organized as follows. Section 2 describes the system model. In Sec. 3, firstly, we model the EE-CSMA/CA mechanism, which takes both the active period and the inactive period into consideration. Secondly, we derive a probability distribution of the service time and the access delay. Thirdly, we analyze the delay jitter of packets and obtain the probability distribution of delay jitter. Finally, we estimate the minimum duration of the active period constrained by access delay or delay jitter through the dichotomy, respectively. Simulations are presented in Sec. 4. We conclude this paper in Sec. 5. Table 1 shows the notations used in this paper and their descriptions.

\section{System Model}

In this paper, we consider the unsaturated system which contains $M$ nodes and one access point (AP). Time 


\begin{tabular}{|c|c|}
\hline Notation & Description \\
\hline$M$ & The number of nodes \\
\hline macMinBE & The minimum backoff exponent \\
\hline $\operatorname{macMaxBE}$ & The maximum backoff exponent \\
\hline$\overline{W_{i}}$ & The contention window size at the $i$-th backoff stage \\
\hline$m$ & The maximum value of backoff \\
\hline$n$ & The maximum value of retransmission \\
\hline$\overline{T_{1}}$ & The duration of active period \\
\hline$T_{2}$ & The duration of inactive period \\
\hline$T$ & The duration of frame structure \\
\hline$R$ & The ratio of active period duration to frame duration \\
\hline$T T_{\mathrm{p}}$ & The required time to transmit a packet \\
\hline$P_{\mathrm{AI}}$ & $\begin{array}{l}\text { The transition probability from the active period to the } \\
\text { inactive period }\end{array}$ \\
\hline$P_{\mathrm{IA}}$ & $\begin{array}{l}\text { The transition probability from the inactive period to } \\
\text { the active period }\end{array}$ \\
\hline$P_{\mathrm{II}}$ & The transition probability of staying in inactive period \\
\hline$w$ & $\begin{array}{l}\text { The probability that the node attempts to the first clear } \\
\text { channel assessment (CCA) }\end{array}$ \\
\hline$\alpha$ & $\begin{array}{l}\text { The probability that a channel is sensed busy when } \\
\text { a node performs the first CCA }\end{array}$ \\
\hline$\beta$ & $\begin{array}{c}\text { The probability that a channel is sensed busy when } \\
\text { a node performs the second CCA }\end{array}$ \\
\hline$\lambda$ & Average arrival rate of packets \\
\hline$l_{0}$ & The probability of no packets in the queue \\
\hline$D_{k}$ & The delay of the $k$-th packet \\
\hline$\overline{D_{k}^{\mathrm{s}}}$ & The needed time to serve the $k$-th packet \\
\hline$J_{k}$ & The delay jitter of the $k$-th packet \\
\hline
\end{tabular}

Tab. 1. Notations and descriptions.

is divided into slots with the equal duration. The length of packets is constant and occupies integer number of slots. The packets can only be transmitted at the beginning of a slot.

The wireless network employs the EE-CSMA/CA for channel access. Figure 1 depicts the frame structure, which includes active period and inactive period. During the active period, nodes adopt CSMA/CA to transmit packets. For the sake of energy efficiency, nodes don't sense the channel during the backoff process, and sense the channel only when the backoff time counter is zero. Nodes stay in sleep mode during the inactive period. During the inactive period, the backoff time counters of all nodes are frozen. Nodes resume their backoff processes in the next active period. In this paper, we focus on analyzing the impact of EE-CSMA/CA mechanism on the access delay and delay jitter. To simplify the problem, we have the assumptions: nodes can hear each other, hence the hidden node problem is avoided; channel is ideal, so packets are error free.

The EE-CSMA/CA mechanism is introduced in Tab. 2. In Tab. 2, $i(0 \leq i \leq m)$ denotes the backoff stage, $r$ $(0 \leq r \leq n)$ represents the retransmission times. The macMinBE and macMaxBE are the minimum and maximum backoff exponent $(B E)$, respectively. $W_{0}$ is the initial backoff window size, where $W_{0}=2^{\text {macMinBE }}$.

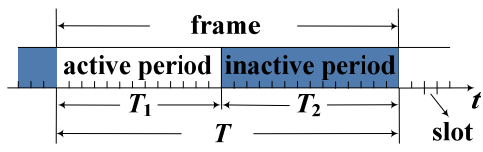

Fig. 1. The frame structure.

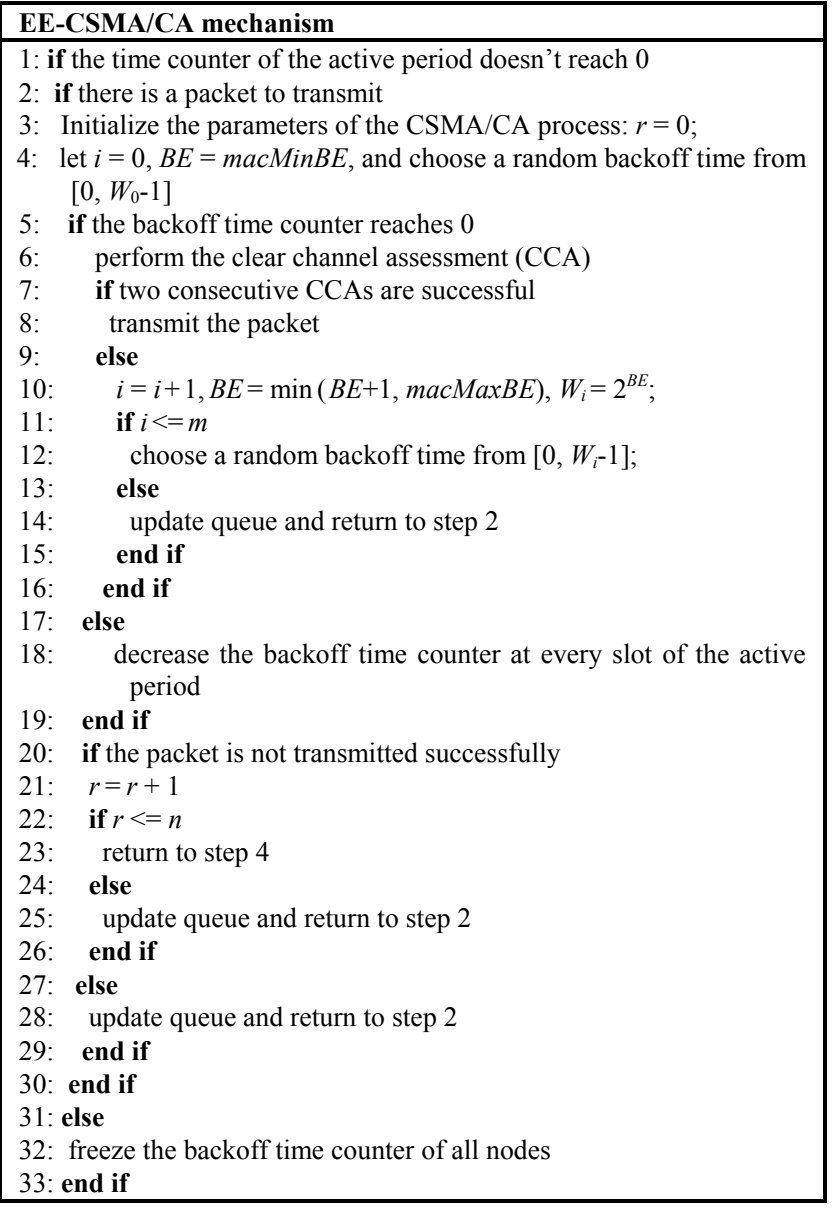

Tab. 2. EE-CSMA/CA mechanism.

\section{Performance Analysis}

\subsection{The Markov Chain Model}

We propose a four-dimensional Markov chain to model the EE-CSMA/CA mechanism, where each state is represented by the four variable $\{s(t), b(t), c(t), h(t)\}$, as shown in Fig. 2. Here, $s(t)$ is defined as the stochastic process representing the backoff stage $i(0 \leq i \leq m)$ of a given node. When the node accesses the channel unsuccessfully, the backoff stage of node increases. $b(t)$ is the stochastic process representing the backoff time counter $j\left(-2 \leq j \leq W_{i}-1\right)$. The backoff time counter is decreased by 1 during each slot of an active period. When the backoff time counter is decreased to zero, a node senses the channel. The states $j=-1$ and $j=-2$ stand for the first CCA and the second CCA, respectively. Considering the retransmission, $c(t)$ represents the value of retransmission $r(0 \leq r \leq n)$. $h(t)$ represents the active or inactive period of the frame structure, where $f=0$ and $f=-1$ stand for the active period and inactive period, respectively.

In this Markov chain, the one-step transition probabilities are calculated as follows. Based on the EECSMA/CA mechanism, when $j \in\left[2, W_{i}-1\right]$, nodes jump to 


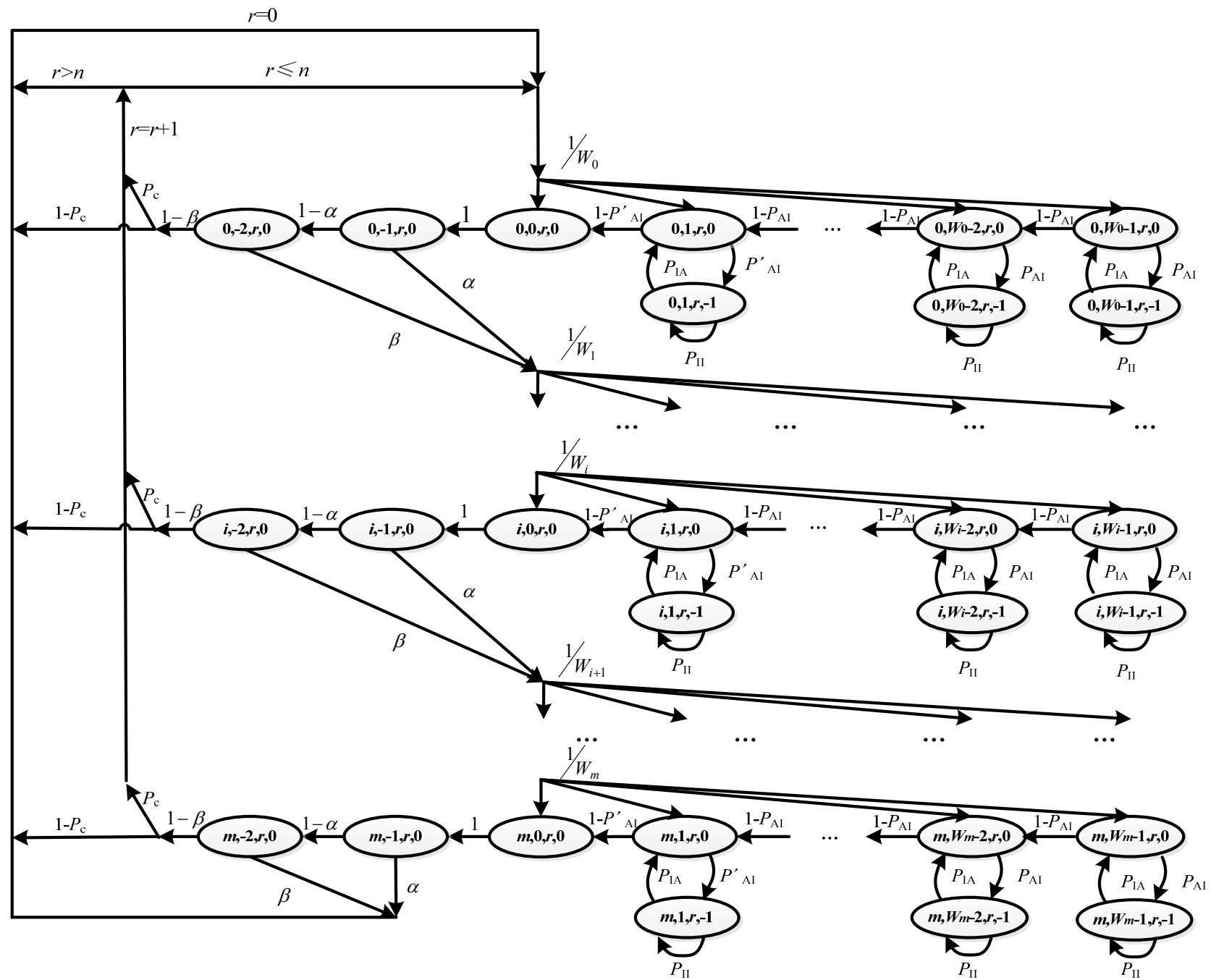

Fig. 2. The four-dimensional Markov chain model.

$b_{0,0,0,0}$
$\left\{\begin{array}{l}\left(\frac{1-g^{n+1}}{1-g}\left(\left(\frac{1-x^{m+1}}{1-x}\right)(3-\alpha)+\left(1+\frac{P_{\mathrm{AI}}^{\prime}}{1-P_{\mathrm{II}}}\right) \frac{1}{1-P_{\mathrm{AI}}^{\prime}}\left(\frac{1-x^{m+1}}{1-x}-\frac{1-(x / 2)^{m+1}}{1-\left(\frac{x}{2}\right)} \frac{1}{W_{0}}\right)+\left(1+\frac{P_{\mathrm{AI}}}{1-P_{\mathrm{II}}}\right) \frac{1}{1-P_{\mathrm{AI}}}\left(\frac{W_{0}}{2} \frac{1-(2 x)^{m+1}}{1-2 x}-\frac{3}{2} \frac{1-x^{m+1}}{1-x}+\frac{1-(x / 2)^{m+1}}{1-(x / 2)} \frac{1}{W_{0}}\right)\right)\right)^{-1} \quad m \leq s \\ \left(\frac{1-g^{n+1}}{1-g}\left(\left(\frac{1-x^{m+1}}{1-x}\right)(3-\alpha)+\left(1+\frac{P_{\mathrm{AI}}^{\prime}}{1-P_{\mathrm{II}}}\right) \frac{1}{1-P_{\mathrm{AI}}^{\prime}}\left(A-\frac{B}{W_{0}}+\left(1-\frac{1}{2^{s} W_{0}}\right) C\right)+\left(1+\frac{P_{\mathrm{AI}}}{1-P_{\mathrm{II}}}\right) \frac{1}{1-P_{\mathrm{AI}}}\left(\frac{W_{0}}{2} \frac{1-(2 x)^{s+1}}{1-2 x}-\frac{3}{2} A+\frac{B}{W_{0}}+\left(\frac{2^{s} W_{0}}{2}-\frac{3}{2}+\frac{1}{2^{s} W_{0}}\right) C\right)\right)\right)^{-1} m>s\end{array}\right.$

the inactive period if the time counter of the active period reaches zero. However, when $j=1$, nodes have to avert the packet transmission and jump to the inactive period if the time counter of the active period has reached a value which is lower than the required time to transmit a packet. Hence, the transition probability of achieving inactive period from the active period to is given by

$$
P\{i, j, r,-1 \mid i, j, r, 0\}= \begin{cases}P_{\mathrm{AI}} & i \in[0, m], j \in\left[2, W_{i}-1\right], r \in[0, n] \\ P_{\mathrm{AI}}^{\prime} & i \in[0, m], j=1, r \in[0, n]\end{cases}
$$

where $P_{\mathrm{AI}}=1 / T_{1}, P_{\mathrm{AI}}^{\prime}=T_{\mathrm{p}} / T_{1} . T_{1}$ and $T_{2}$ denote the duration of active period and inactive period, respectively. $T_{\mathrm{p}}$ is the required time to transmit a packet.
Due to the decrement of backoff time counter, the transition probabilities are given by

$$
P\{i, j, r, 0 \mid i, j+1, r, 0\}= \begin{cases}1-P_{\mathrm{AI}} & i \in[0, m], j \in\left[1, W_{i}-2\right], r \in[0, n] \\ 1-P_{\mathrm{AI}}^{\prime} & i \in[0, m], j=0, r \in[0, n]\end{cases}
$$

Let $P_{\mathrm{IA}}$ be the transition probability from the inactive period to the active period and $P_{\mathrm{II}}$ be the transition probability of staying in the inactive period, then

$$
\begin{aligned}
& P\{i, j, r, 0 \mid i, j, r,-1\}=P_{\mathrm{IA}} \quad i \in[0, m], j \in\left[1, W_{i}-1\right], r \in[0, n], \\
& P\{i, j, r,-1 \mid i, j, r,-1\}=P_{\mathrm{II}} \quad i \in[0, m], j \in\left[1, W_{i}-1\right], r \in[0, n]
\end{aligned}
$$

where $P_{\mathrm{IA}}=T_{1} /\left(T_{1}+T_{2}\right)$ and $P_{\mathrm{II}}=1-P_{\mathrm{IA}}$. 
Other transition probabilities of Markov chain are shown in Appendix A.

Let $b_{i, j, r, f}$ be the probability of the steady state $(i, j, r, f)$ of the Markov chain. From Fig. 2, we obtain

$$
\begin{gathered}
b_{i, 0, r, 0}=(\alpha+(1-\alpha) \beta) b_{i-1,0, r, 0}, \\
b_{0,0, r, 0}=(1-\alpha)(1-\beta) P_{\mathrm{c}} \sum_{i=0}^{m} b_{i, 0, r-1,0} .
\end{gathered}
$$

For convenience, let $x=\alpha+(1-\alpha) \beta$ in this paper, then we rewrite $b_{i, 0, r, 0}=(\alpha+(1-\alpha) \beta) b_{i-1,0, r, 0}$ as $b_{i, 0, r, 0}=x^{i} b_{0,0, r, 0}$. Let $g=P_{\mathrm{c}}\left(1-x^{m+1}\right)$, so

$$
b_{0,0, r, 0}=(1-x) P_{\mathrm{c}} \frac{1-x^{m+1}}{1-x} b_{0,0, r-1,0}=g^{r} b_{0,0,0,0} .
$$

Owing to the Markov chain regularities, we have

$$
\begin{gathered}
b_{i, j, r, 0}=\frac{W_{i}-j}{W_{i}} \frac{b_{i, 0, r, 0}}{1-P_{\mathrm{AI}}} \quad i \in[0, m], j \in\left[2, W_{i}-1\right], r \in[0, n], \\
b_{i, 1, r, 0}=\frac{W_{i}-1}{W_{i}} \frac{b_{i, 0, r, 0}}{1-P_{\mathrm{AI}}^{\prime}} \quad i \in[0, m], j=1, r \in[0, n] .
\end{gathered}
$$

According to (8) and (9), $b_{i, j, r, f}$ can be expressed as functions of $b_{0,0,0,0}$. The sum of the steady state probabilities of the Markov chain is 1 . It can be expressed as

$$
\sum_{i=0}^{m} \sum_{j=0}^{W_{i}-1} \sum_{r=0}^{n} b_{i, j, r, 0}+\sum_{i=0}^{m} \sum_{j=1}^{W_{i}-1} \sum_{r=0}^{n} b_{i, j, r,-1}+\sum_{i=0}^{m} \sum_{j=-2}^{-1} \sum_{r=0}^{n} b_{i, j, r, 0}=1 .
$$

Using (5), (6), (8) and (9), equation (10) can be expanded. So $b_{0,0,0,0}$ is given by (11) (below Fig. 2). In (11), $A=\left(1-x^{s+1}\right) /(1-x), \quad B=\left(1-(x / 2)^{s+1}\right) /(1-(x / 2)), \quad C=$ $\left(x^{s+1}-x^{m+1}\right) /(1-x)$ and $s=\operatorname{macMaxBE}-\operatorname{macMinBE}$.

$w$ is defined as the probability that the node attempts to the first CCA in any slot. In other words, $w$ denotes the probability of the backoff time counter of the node being zero in the slot.

$$
w=\sum_{i=0}^{m} \sum_{r=0}^{n} b_{i, 0, r, 0}=\frac{1-x^{m+1}}{1-x} \frac{1-g^{n+1}}{1-g} b_{0,0,0,0} .
$$

$\alpha$ represents the probability that a channel is sensed busy when the node attempts to the first CCA, that is, at least one of the others is transmitting in the current slot. When a channel is sensed idle for the first CCA, the second CCA will be carried out.

$$
\alpha=T_{\mathrm{p}}\left(1-\left(1-\left(1-l_{0}\right) w\right)^{M-1}\right)(1-\alpha)(1-\beta) .
$$

Let $\beta$ stands for the probability that a channel is sensed busy when the node performs the second CCA. The channel is sensed busy for the second CCA, that means, at least one of the others has been carried out the second CCA when the node performs the first CCA.

$$
\beta=\left(1-\left(1-\left(1-l_{0}\right) w\right)^{M-1}\right)(1-\beta)
$$

where $l_{0}$ is the probability of no packets in the queue. Referring to [22], we model the buffer of a node as a queuing system. The number of packets in the queue is characterized by a discrete time Markov chain. Based on the Markov theory, we obtain the steady state probability of the Markov chain in Appendix B. Hence, $l_{0}$ is obtained.

By the given equations, we can obtain $w, \alpha, \beta, b_{0,0,0,0}$. Using the above definitions, we get the probability of collision as

$$
P_{\mathrm{c}}=\left(1-\left(1-\left(1-l_{0}\right) w\right)^{M-1}\right)(1-\alpha)(1-\beta) .
$$

The probability of successful transmission is expressed as

$$
P_{\mathrm{s}}=1-P_{\mathrm{c}}
$$

\subsection{The Service Time and the Average Access Delay}

\section{A. The Service Time Distribution}

In this section, we calculate the service time distribution of packets in probability generation domain. The service time distribution of packets cannot be solved in the probability domain. Since the service time is a non-negative random variable, we analyze the service time relying on our derivation of its probability generation function (PGF). The PGF of the service time of a packet is

$$
G(z)=\sum_{D_{k}^{\mathrm{s}}=0}^{+\infty} p\left(D_{k}^{\mathrm{s}}\right) z^{D_{k}^{\mathrm{s}}}
$$

where $p\left(D_{k}^{\mathrm{s}}\right)$ is the probability that the service time of packet $k$ is $D_{k}^{\mathrm{s}}$.

In the backoff process, the backoff time counter either decreases by 1 with the probability $1-P_{\mathrm{AI}}$ or jumps to the inactive period from the active period with the probability $P_{\mathrm{AI}}$. Let $H_{d}^{j}(z)$ be the PGF of time interval needed in decreasing the backoff time counter by 1 . Then we obtain

$$
H_{d}^{j}(z)=\left\{\begin{array}{lc}
P_{\mathrm{AI}} z^{T_{2}}+\left(1-P_{\mathrm{AI}}\right) z & i \in[0, m], j \in\left[2, W_{i}-1\right] \\
P_{\mathrm{AI}}^{\prime} z^{T_{2}}+\left(1-P_{\mathrm{AI}}^{\prime}\right) z & i \in[0, m], j=1
\end{array}\right.
$$

$H_{i}(z)$ is the PGF of required time that the backoff time counter decreases to zero when the backoff stage is $i$. $H_{i}(z)$ is

$H_{i}(z)= \begin{cases}\frac{1}{W_{i}}\left[1+H_{d}^{1}(z)+H_{d}^{1}(z) H_{d}^{2}(z)+\cdots+\Pi_{j=1}^{W_{i}-1} H_{d}^{j}(z)\right] & i \leq s \\ \frac{1}{W_{s}}\left[1+H_{d}^{1}(z)+H_{d}^{1}(z) H_{d}^{2}(z)+\cdots+\Pi_{j=1}^{W_{s}-1} H_{d}^{j}(z)\right] & i>s\end{cases}$

After the backoff process, the node attempts to access the channel. Within a retransmission, $T(z)$ is the PGF of the required time that the node successfully accesses the channel.

$$
\begin{aligned}
& T(z)= \\
& (1-\alpha)(1-\beta) D^{2}(z) \sum_{i=0}^{m}\left(\left(\alpha D(z)+(1-\alpha) \beta D^{2}(z)\right)^{i} \prod_{q=0}^{i} H_{q}(z)\right)
\end{aligned}
$$


where $D(z)$ is the PGF of the required time (1 slot) for a CCA detection.

$$
D(z)=z .
$$

Considering the maximum value of retransmission, let $F(z)$ is the PGF of the required time that the node unsuccessfully accesses the channel, and the reason for the failure is that the node has reached the maximum value of backoff within a retransmission.

$$
F(z)=\left(\alpha D(z)+(1-\alpha) \beta D^{2}(z)\right)^{m+1} \prod_{i=0}^{m} H_{i}(z)
$$

According to the equations and the EE-CSMA/CA mechanism, $G(z)$ is obtained

$$
\begin{aligned}
G(z)= & P_{\mathrm{s}} T(z) S(z) \sum_{i=0}^{n}\left(T(z)\left(1-P_{\mathrm{s}}\right) C(z)\right)^{i}+\left(T(z)\left(1-P_{\mathrm{s}}\right) C(z)\right)^{n+1} \\
& +F(z) \sum_{i=0}^{n}\left(T(z)\left(1-P_{\mathrm{s}}\right) C(z)\right)^{i}
\end{aligned}
$$

where $S(z)$ and $C(z)$ are the PGFs of the required time for a successful and unsuccessful transmission of a packet, respectively.

$$
S(z)=z^{T_{\mathrm{p}}}, C(z)=z^{T_{\mathrm{p}}} .
$$

\section{B. The Average Access Delay}

The average access delay is the mathematical expectation of the service time. The probability distribution of the service time is derived by the inverse transform. It is a general distribution and can be expressed as

$$
p_{\text {service }}(t)=\sum_{D_{k}^{\mathrm{s}}=0}^{+\infty} p\left(D_{k}^{\mathrm{s}}\right) \delta\left(t-D_{k}^{\mathrm{s}}\right) .
$$

Here, $\delta(t)$ is the unit impulse function.

Then, the average access delay are obtained as

$$
E\left[D_{k}^{\mathrm{s}}\right]=\sum_{D_{k}^{\mathrm{s}}=0}^{+\infty} D_{k}^{\mathrm{s}} p\left(D_{k}^{\mathrm{s}}\right) .
$$

\subsection{The Analysis of Delay Jitter}

The delay jitter is a QoS metric, which is defined as the difference of delay experienced by two successive packets. So delay jitter is showed as

$$
J_{k}=D_{k}-D_{k-1}
$$

where $D_{k}$ is the delay of packet $k$. The delay $D_{k}$ contains both the waiting delay and the service delay. The packets in the queue are served in accordance with the principle of first-in-first-out (FIFO). The waiting delay is defined as the time interval between the arrival moment of the packet and the moment when the packet starts to be served. The service delay is the time to serve a packet. In other words, the service delay is the service time of the packets. For CSMA/CA, the service delay of a packet is random. The arrival moment of packets is random, so delay derivation is a hard task. As the difference in delay, delay jitter is an even hard issue to be handed. We analyze the delay jitter by a new approach.

For the analysis of delay jitter, we analyze the delay jitter of packets from two situations, which are the empty case and the non-empty case, respectively. In this paper, the definition of delay jitter is not the absolute value of delay jitter. Therefore, the delay jitter could be a negative value.

\section{A. The Case of Empty Queue}

When the queue is empty, i.e. there is no packet in the queue, packets can be served immediately after arriving. Hence the delay jitter can be expressed as

$$
J_{k}=D_{k}^{\mathrm{s}}-D_{k-1}^{\mathrm{s}}
$$

where $D_{k}^{\mathrm{s}}$ is the service time of packet $k$.

As a result, the statistics of $J_{k}$ is defined by the joint distribution of two sequential service times $p_{2 s}\left(D_{k}^{\mathrm{s}}, D_{k-1}{ }^{\mathrm{s}}\right)$. Based on the statistics, the distribution of difference of two random variables is given as

$$
p_{\text {empty }}\left(J_{k}\right)=\int_{-\infty}^{+\infty} p_{2 s}\left(D_{k-1}^{\mathrm{s}}+J_{k}, D_{k-1}^{\mathrm{s}}\right) \mathrm{d} D_{k-1}^{\mathrm{s}} .
$$

According to the EE-CSMA/CA mechanism, the node initializes all parameters after the transmission of a packet. Then, the next packet of the node restarts the competition for the channel. Therefore, we can know that the distribution of service time for packets is independent. So (29) is rewritten as

$$
p_{\text {empty }}\left(J_{k}\right)=\int_{-\infty}^{+\infty} p_{\text {service }}\left(D_{k-1}^{s}+J_{k}\right) p_{\text {service }}\left(D_{k-1}^{\mathrm{s}}\right) \mathrm{d} D_{k-1} \mathrm{~s} .
$$

In the subsection B of Sec. 3.2, we obtain the service time distribution of packets. Using the probability distribution of service time, the probability distribution of the delay jitter is given for the empty queue as

$$
p_{\text {empty }}\left(J_{k}\right)=\sum_{D_{k-1}=0}^{+\infty} p\left(D_{k-1}^{\mathrm{s}}+J_{k}\right) p\left(D_{k-1}^{\mathrm{s}}\right) .
$$

\section{B. The Case of Non-Empty Queue}

The situation that the queue is non-empty means that there are packets in the queue. It is assumed that packet $k-1$ arrives at the queue at $t_{0}\left(t_{0}<0\right)$ and starts being served at $t=0$. Packet $k$ arrives at the queue at $t_{0}+\tau$ and starts being served at $D_{k-1}^{\mathrm{s}}$, as shown in Fig. 3. For the case of the non-empty queue, the packet $k$ has already been in the queue when the packet $k-1$ begins to be served. Here, $\tau$ is the inter-arrival time of two sequential packets. The packet $k-1$ and packet $k$ leave the queue at the $D_{k-1}{ }^{\mathrm{s}}$ and $D_{k-1}{ }^{\mathrm{s}}+D_{k}^{\mathrm{s}}$, respectively. Therefore, the delay jitter could be evaluated as

$$
\begin{aligned}
J_{k} & =D_{k}-D_{k-1} \\
& =\left(D_{k}^{\mathrm{s}}+D_{k-1}^{\mathrm{s}}-t_{0}-\tau\right)-\left(D_{k-1}^{\mathrm{s}}-t_{0}\right) \\
& =D_{k}^{\mathrm{s}}-\tau .
\end{aligned}
$$




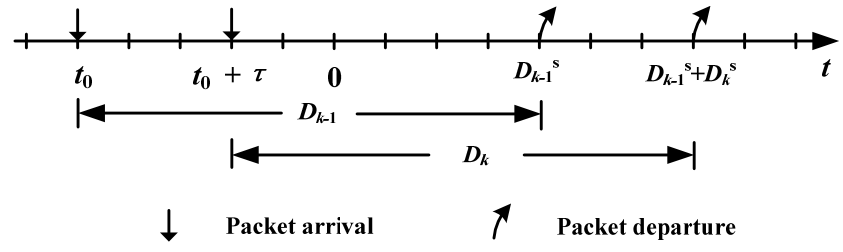

Fig. 3. Queuing analysis.

Delay jitter is related to the inter-arrival time of two sequential packets and the service time of the packet. They are independent, so the probability distribution of delay jitter for the non-empty queue is shown as

$$
p_{\text {non-empty }}\left(J_{k}\right)=\int_{-\infty}^{+\infty} p_{\text {service }}\left(\tau+J_{k}\right) p_{\mathrm{A}}(\tau) \mathrm{d} \tau
$$

where $p_{\mathrm{A}}(\tau)$ is the distribution of the inter-arrival time of two sequential packets. So we can derive delay jitter for the non-empty queue

$$
p_{\text {non-empty }}\left(J_{k}\right)=\sum_{D_{k}^{\mathrm{s}}=0}^{+\infty} p\left(D_{k}^{\mathrm{s}}\right) p_{\mathrm{A}}\left(D_{k}^{\mathrm{s}}-J_{k}\right) .
$$

\section{The General Case}

In the general case, the queue of node is sometimes empty and sometimes not empty. Let $l_{0}$ be the probability that the queue is empty. According to the previous analysis, the distribution of delay jitter for the general case can be expressed as follows

$$
p_{\text {general }}\left(J_{k}\right)=l_{0} p_{\text {empty }}\left(J_{k}\right)+\left(1-l_{0}\right) p_{\text {non-empty }}\left(J_{k}\right) .
$$

From Appendix B, $l_{0}$ is obtained.

The average delay jitter is defined as expectation of the absolute value of the delay jitter, which is expressed as

$$
E\left[\left|J_{k}\right|\right]=\sum_{J_{k}=-\infty}^{+\infty}\left|J_{k}\right| p_{\text {general }}\left(J_{k}\right)
$$

\subsection{The Minimum Duration of the Active Period Constrained by QoS}

In this section, we estimate the minimum duration of the active period constrained by access delay and delay jitter by the dichotomy algorithm, respectively. We have obtained the access delay and the delay jitter in the previous analysis. Different durations of the active period and inactive period have influence on the access delay and the delay jitter. For energy saving and QoS guarantee, it is necessary to design an algorithm to estimate the minimum duration of the active period. The duration of the frame structure is constant and the active period contains integer number of slots. For the simplicity of the algorithm, we adopt the dichotomy method to solve this problem. Considering the access delay constraint, we formulate the optimization problem as

$$
\begin{aligned}
& \min T_{1} \\
& \text { s.t. } E\left[D_{k}^{\mathrm{s}}\right] \leq \text { Delay }
\end{aligned}
$$

where Delay is the target of the access delay.

For the delay jitter constraint, the optimization problem is formulated as

$$
\begin{aligned}
& \min T_{1} \\
& \text { s.t. } E\left[\left|J_{k}\right|\right] \leq \text { Jitter }
\end{aligned}
$$

where Jitter denotes the target of the delay jitter.

In this paper, we adopt the dichotomy algorithm to deal with two optimization problems. As an example, we present the pseudo-code of the dichotomy with access delay constraint in Tab. 3 .

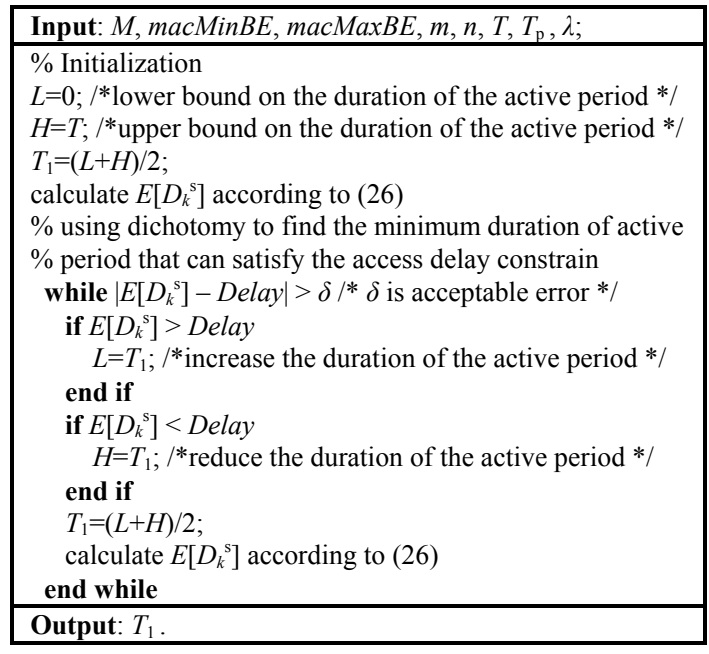

Tab. 3. The dichotomy algorithm.

\section{Simulations and Results Analysis}

In this section, we present the theoretical results and simulated results of access delay and delay jitter. The simulated results are obtained using the MATLAB, where we simulate the EE-CSMA/CA mechanism. And then we calculate the probability distribution of service delay and delay jitter. The parameters used in the theoretical results and simulated results are listed in Tab. 4. We consider the arrival of packets is the Poisson distribution. The CSMA/CA mechanism with inactive period is suitable for a system with light load. Packets arrive at nodes with the average rate $\lambda$ (the number of packets per slot).

\begin{tabular}{|c|c|}
\hline The number of nodes $M$ & 5 \\
\hline Initial backoff window $W_{0}$ & 8 \\
\hline macMinBE & 3 \\
\hline macMaxBE & 5 \\
\hline The maximum backoff stage $m$ & 4 \\
\hline $\begin{array}{c}\text { The maximum retransmission } \\
\text { times } n\end{array}$ & 3 \\
\hline Packet payload & 4000 bits \\
\hline MAC header & 272 bits \\
\hline PHY header & 120 bits \\
\hline Data bit rate & $54 \mathrm{Mbit} / \mathrm{s}$ \\
\hline Slot time & $20 \mu \mathrm{s}$ \\
\hline
\end{tabular}

Tab. 4. Parameters used in the simulations. 
Figure 4 presents the service time distribution of packets in several cases. Both the durations of active and inactive period in the frame structure are set as 50 slots in Fig. 4. It is found that the service time of packets grows along with the arrival rate of packets. The arrival rate of packets increases, the competition in the networks is fierce. The probability that the channel is busy and the collision probability of a packet increase, which results in the increase of the times of backoff or the times of retransmission. Therefore, the service time of packets increases. It is observed that the service time of packets may be around 60 slots in Fig. 4. The reason is that the packet is not served completely in the current active period, and it waits for an inactive period, then continuously served in the next active period. The theoretical results and simulated results are basically the same in Fig. 4, but there exist some differences between them. It may be caused by the approximation in modeling. It is assumed that service process could enter the inactive state from any states of backoff operation with the same transition probability. We are interesting in refining the model in our next step study.

Figure 5 shows the average access delay of packets under the different number of nodes and various frame structures. The average access delay goes up along with the number of nodes. Meanwhile, it is presented that the average access delay goes down as the active ratio $R$ increases. When the duration of active period becomes bigger, nodes may be served within the current active period. Therefore, the performance of average access delay is better. In contrast to the CSMA/CA mechanism with the inactive period, the conventional CSMA/CA mechanism has better access delay performance at the cost of energy consumption. The three curves bound by the dotted circle are the case that the active ratio $R$ is set to 0.5 . In this case, their performance of average access delay is similar.

Figure 6 presents the probability distribution of the delay jitter. Both the durations of active and inactive period in the frame structure are set as 50 slots in Fig. 6 . When the arrival rate is small, the theoretical results and simulated results are basically the same. There is a slight gap between the theoretical results and the simulated results when the arrival rate is big. When the arrival rate of packets is the lower, the values of delay jitter are small and centered around 0,50 and -50 . In this situation, since no packets are waiting in the queue. The delay jitter is caused by the difference between the service time of two consecutive packets. As shown in Fig. 4, with a higher probability, the service time of packets takes 10 and 60 slots. As a result, the absolute value of delay jitter takes 0 and 50 slots with a higher probability. When the arrival rate of packets is the higher, the queue of nodes is not empty. It is found that the performance of delay jitter gets worse. In fact, when the traffic of services is large, the performance of delay jitter is poor. It can be seen that the service delay of packets increases with the arrival rate of packets from Fig. 4 . The performance of delay jitter deteriorates. Values of delay jitter cover a big space of values.

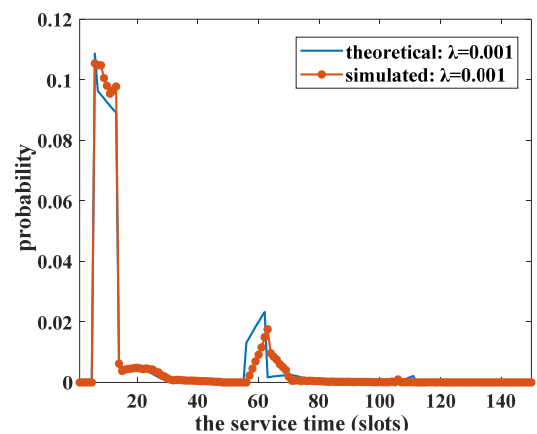

(a) The probability distribution of service time $(\lambda=0.001)$

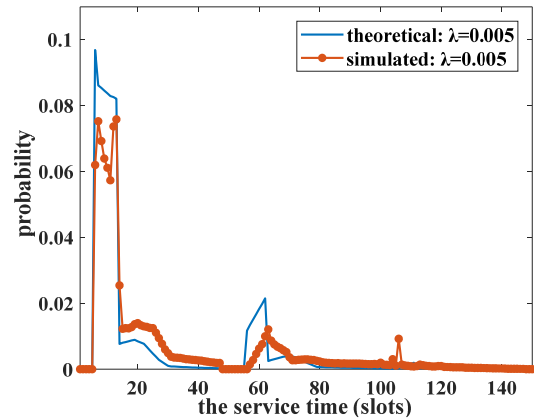

(b) The probability distribution of service time $(\lambda=0.005)$.

Fig. 4. The probability distribution of service time under different arrival rates.

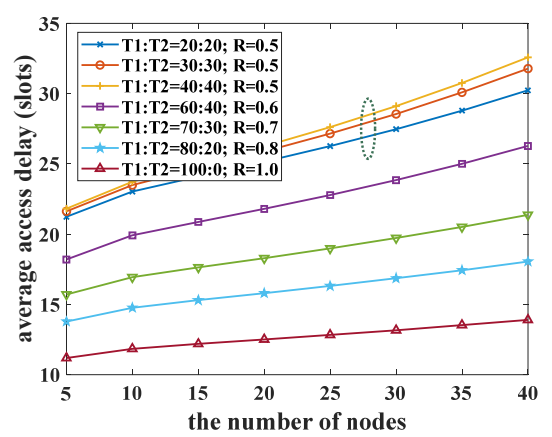

Fig. 5. The average access delay versus the number of nodes and the frame structure $(\lambda=0.003)$.

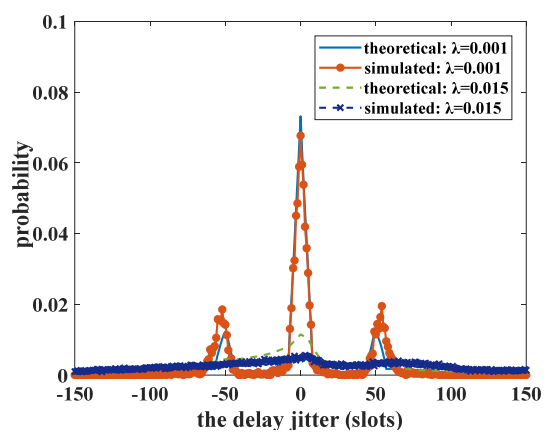

Fig. 6. The probability distribution of delay jitter.

Figure 7 shows the impacts of the number of nodes and the several frame structures on the average delay jitter. It is obvious that the average delay jitter rises with the number of nodes. When the active ratio $R$ increases, the performance of delay jitter gets better. When the duration 
of active period increases and the duration of frame remains unchanged, the service time of packets shortens. Further, the performance of access delay gets better, and then average delay jitter decreases. The longer the active period is, the better the delay jitter performance is, but the more energy consumption is. The three curves bound by the dotted circle are the case that the active ratio $R$ is set to 0.5 . When the active ratio $R$ is set to be 0.5 , the average delay jitter increases along with the duration of the active period. That is, the duration of the active period affects the delay jitter when the active ratio $R$ is constant.

In Fig. 5 and Fig. 7, it is presented that the access delay and the delay jitter vary with configurations of the frame structure. Under the access delay or delay jitter constraints (25 slots), we estimate the suitable duration of the active period by the dichotomy in Fig. 8. Under different number of nodes and arrival rate of packets, we show the suitable duration of the active period constrained by the access delay in Fig. 8(a), and constrained by the delay jitter

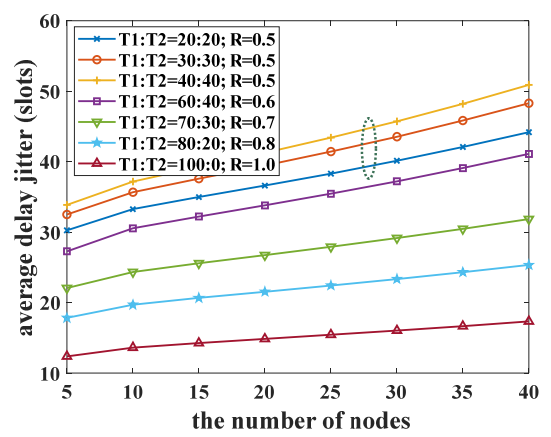

Fig. 7. The average delay jitter versus the number of nodes and the frame structure $(\lambda=0.003)$

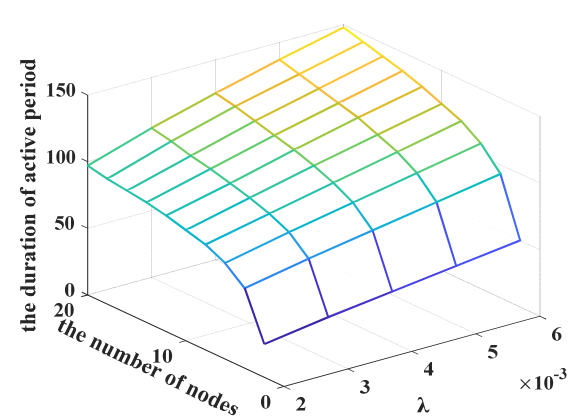

(a) The duration of active period under access delay constraint.

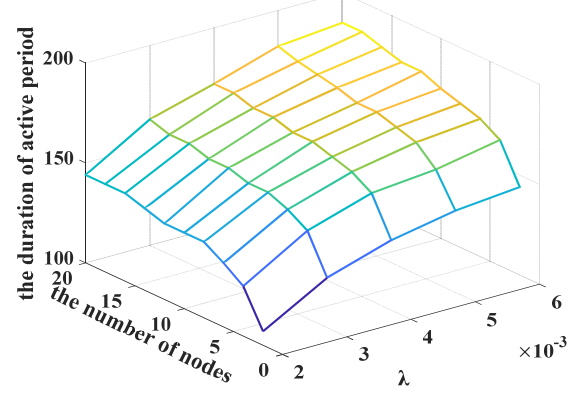

(b) The duration of active period under delay jitter constraint.

Fig. 8. The duration of active period constrained by QoS. in Fig. 8(b). A frame is set to be 200 slots in Fig. 8. With the increase of the arrival rate of packets or the number of nodes, the service time of packets increase. The performance of access delay and delay jitter degrades. To ensure the access delay or delay jitter constraint, the duration of the active period increases. It means that the more nodes or the higher arrival rate are, the longer the needed active period is.

\section{Conclusion}

In this paper, we modeled the CSMA/CA mechanism with the consideration of its active period and inactive period. We derived the probability distribution of the service time in probability generation domain. Then, we decoupled the queue in a node and configured two logical queues, which helped to enable the complex analysis of delay jitter. Queuing theory based analysis indicated that the delay jitter was associated with the arrival and the service. Based on the whole probability analysis, the probability distribution of the delay jitter is obtained. The simulation results showed that both of the duration of active period and inactive period (i.e., frame structure) had effect on the access delay and the delay jitter. For energy saving and QoS guarantee, we found the suitable duration of the active period by the dichotomy under the constraints of access delay or delay jitter. It is interesting to explore the delay jitter of real-time service in the networks, especially when it coexists with other several classes of services. This is our next research subject.

\section{Appendix A}

Considering the random binary exponential backoff process, the contention window size at the $i$-th stage is shown as follows:

$$
W_{i}= \begin{cases}2^{i} W_{0} & i \leq s \\ 2^{s} W_{0} & i>s\end{cases}
$$

where $W_{0}=2^{\text {macMinBE }}, s=\operatorname{macMaxBE}-\operatorname{macMin} B E$. And parts of transition probabilities are shown as follows.

$$
\begin{aligned}
P\{i+1, j, r, 0 \mid i,-1, r, 0\} & =\frac{\alpha+(1-\alpha) \beta}{W_{i+1}}, \\
i & \in[0, m-1], j \in\left[0, W_{i+1}-1\right], r \in[0, n], \\
P\{0, j, 0,0 \mid i,-1, r, 0\} & =\frac{(1-\alpha)(1-\beta)\left(1-P_{\mathrm{c}}\right)}{W_{0}}, \\
i & \in[0, m], j \in\left[0, W_{0}-1\right], r \in[0, n-1], \\
P\{0, j, 0,0 \mid i,-1, n, 0\} & =\frac{(1-\alpha)(1-\beta)}{W_{0}}, \\
i & \in[0, m-1], j \in\left[0, W_{0}-1\right], r=n, \\
P\{0, j, 0,0 \mid m,-1, n, 0\} & =\frac{1}{W_{0}}, \quad i=m, j \in\left[0, W_{0}-1\right], r=n,
\end{aligned}
$$




$$
\begin{aligned}
& P\{0, j, r+1,0 \mid i,-1, r, 0\}=\frac{(1-\alpha)(1-\beta) P_{\mathrm{c}}}{W_{0}}, \\
& i \in[0, m], j \in\left[0, W_{0}-1\right], r \in[0, n-1], \\
& P\{0, j, 0,0 \mid m,-1, r, 0\}=\frac{\alpha+(1-\alpha) \beta}{W_{0}}, \\
& i=m, j \in\left[0, W_{0}-1\right], r \in[0, n-1], \\
& P\{i,-1, r, 0 \mid i, 0, r, 0\}=1, \quad i \in[0, m], r \in[0, n] .
\end{aligned}
$$

The first equation of (40) stands for the transition probability when the node chooses randomly backoff time counter in the next backoff stage after the failed first CCA or the failed second CCA. The second equation of (40) is the transition probability when the node goes to initial state after transmitting successfully. The third and fourth equations of (40) are the transition probabilities when the node goes to initial state after reaching the maximum value of retransmission. The fifth equation of (40) defines the transition probability when the node goes to the next retransmission after transmitting unsuccessfully. The sixth equation of (40) is the transition probability when the node goes to initial state and chooses randomly backoff time counter after the failed first CCA or the failed second CCA in the maximum value of backoff. The seventh equation of (40) expresses that the node senses a channel when the backoff time counter decreases to zero.

\section{Appendix B}

In this section, we model the queuing system in any node as a $\mathrm{M} / \mathrm{G} / 1 / K$ queue, where $K$ is the capacity of the queue. The number of packets in the queue is a discrete time Markov chain, and the state space of the queuing system is represented as $\{0,1, \ldots, K-1\}$. The transition probability matrix $\mathbf{P}$ is given by

$$
\mathbf{P}=\left[\begin{array}{llllll}
A_{0} & A_{1} & A_{2} & \cdots & A_{K-2} & A_{K-1}^{\prime} \\
A_{0} & A_{1} & A_{2} & \cdots & A_{K-2} & A_{K-1}^{\prime} \\
0 & A_{0} & A_{1} & \cdots & A_{K-3} & A_{K-2}^{\prime} \\
\vdots & \vdots & \vdots & \ddots & \vdots & \vdots \\
0 & 0 & 0 & \cdots & A_{0} & A_{1}^{\prime}
\end{array}\right]
$$

where $A_{i}$ and $A_{i}^{\prime}$ can be shown as follows

$$
\begin{gathered}
A_{i}=\sum_{D_{k}^{\mathrm{s}}=1}^{\infty} a\left(i, D_{k}^{\mathrm{s}}\right) p\left(D_{k}^{\mathrm{s}}\right), \\
A_{i}^{\prime}=1-\sum_{j=0}^{i-1} A_{j}
\end{gathered}
$$

where $a\left(i, D_{k}^{\mathrm{s}}\right)$ represents the probability that there are $i$ packets arriving at the node in the $D_{k}^{\mathrm{s}}$ time interval, and $p\left(D_{k}{ }^{\mathrm{s}}\right)$ is the probability that the service time of packet $k$ is $D_{k}^{\mathrm{s}}$.

The limiting state probability vector of the queue is $\pi=\left(\pi_{0}, \pi_{1}, \ldots, \pi_{K-1}\right)$. Based on the Markov theory, it is obtained. The steady state probability of Markov chain $l_{i}$, the mean delay $T$, and throughput $S$ are as follows

$$
\begin{gathered}
l_{i}=\frac{\pi_{i}}{\lambda E\left[D_{k}^{\mathrm{s}}\right]+\pi_{0}}, \quad 0 \leq i \leq K-1, \\
l_{K}=1-\sum_{0}^{K-1} l_{i}, \quad i=K, \\
T=\frac{1}{\lambda\left(1-l_{K}\right)}\left[\sum_{k=0}^{K-1} k \pi_{k}+K\left(\lambda E\left[D_{k}^{\mathrm{s}}\right]+\pi_{0}-1\right)\right], \\
S=\lambda\left(1-l_{K}\right)\left(1-x^{m+1} \frac{1-g^{n+1}}{1-g} b_{0,0,0,0}\right)\left(1-P_{\mathrm{c}}\left(1-x^{m+1}\right) g^{n} b_{0,0,0,0}\right)
\end{gathered}
$$

In this paper, we apply the iterative algorithm, and the steps are shown as following steps.

(1) Initialize $l_{0}=0$, which is the saturated condition.

(2) Based on $l_{0}$, calculate the $P_{\mathrm{s}}$.

(3) Calculate the service time distribution of the packets in the probability generation domain.

(4) Calculate the steady state probability of the queuing system.

(5) Calculate the mean delay $T$ and throughput $S$.

(6) If $T$ and $S$ converge to the previous value, the algorithm will stop; otherwise, update $l_{0}$ and return step 2 . The algorithm will repeat until the convergence condition is satisfied.

\section{References}

[1] HASSAN, H., KHAN, M. N., GILANI, S. O., et al. H.264 encoder parameter optimization for encoded wireless multimedia transmissions. IEEE Access, 2018, vol. 6, p. 22046-22053. DOI: 10.1109/ACCESS.2018.2824835

[2] KYRBASHOV, B., BARONAK, I., KOVACIK, M., et al. Evaluation and investigation of the delay in VoIP networks. Radioengineering, 2011, vol. 20, no. 2, p. 540-547.

[3] CHEN, K. T., HUANG, C. Y., HUANG, P., et al. Quantifying Skype user satisfaction. ACM Sigcomm Computer Communication Review, 2006, vol. 36, no. 4, p. 399-410. DOI: $10.1145 / 1151659.1159959$

[4] ZHAO, N., CHI, X. F., ZHAO, L. L., et al. A spectrum efficient self-admission framework for coexisting IEEE 802.15.4 networks under heterogeneous traffics. Radioengineering, 2018, vol. 27, no. 1, p. 326-334. DOI: 10.13164/re.2018.0326

[5] KO, Y. M., PENDER, J. Strong approximations for time-varying infinite-server queues with non-renewal arrival and service processes. Stochastic Models, 2018, vol. 34, no. 2, p. 186-206. DOI: 10.1080/15326349.2018.1425886

[6] WU, Y., MIN, G., WANG, G., et al. Modelling of heterogeneous wireless networks under batch arrival traffic with communication locality. In IEEE Wireless Communications and Networking Conference (WCNC). Nevada, 2009, p. 2798-2803. DOI: 10.1109/WCNC.2009.4917810

[7] CHYDZINSKI, A., SAMOCIUK, D., ADAMCZYK, B. Burst ratio in the finite-buffer queue with batch Poisson arrivals. Applied Mathematics and Computation, 2018, vol. 330, p. 225-238. DOI: 10.1016/j.amc.2018.02.021 
[8] BIANCHI, G. Performance analysis of the IEEE 802.11 distributed coordination function. IEEE Journal on Selected Areas in Communications, 2000, vol. 18, no. 3, p. 535-547. DOI: $10.1109 / 49.840210$

[9] DAI, L., SUN, X. H. A unified analysis of IEEE 802.11 DCF networks: stability, throughput, and delay. IEEE Transactions on Mobile Computing, 2013, vol. 12, no. 8, p. 1558-1572. DOI: 10.1109/TMC.2012.128

[10] CHANDRA, K., PRASAD, V., NIEMEGEERS, I. Performance analysis of IEEE 802.11ad MAC Protocol. IEEE Communications Letters, 2017, vol. 21, no. 7, p. 1513-1516. DOI: 10.1109/LCOMM.2017.2677924

[11] BANERJEE, B., MUKHERJEE, A., NASKAR, M. K., et al. BSMAC: A hybrid MAC protocol for IoT systems. In IEEE Global Communications Conference (IEEE GLOBECOM). Washington, 2017, p. 1-7. DOI: 10.1109/GLOCOM.2016.7841643

[12] MALIK, M. H., JAMIL, M., KHAN, M. N., et al. Formal modelling of TCP congestion control mechanisms ECN/RED and SAP-LAW in the presence of UDP traffic. EURASIP Journal on Wireless Communications and Networking, 2016, vol. 156, p. 1-12. DOI: $10.1186 / \mathrm{s} 13638-016-0646-9$

[13] XIAO, Z. L., ZHOU, J., YAN, J. J., et al. Performance evaluation of IEEE 802.15.4 with real time queueing analysis. Ad Hoc Networks, 2018, vol. 73, p. 80-94. DOI: 10.1016/j.adhoc.2018.01.006

[14] PORETSKY, S., PERSER, J., ERRAMILLI, S., et al. Terminology for benchmarking network-layer traffic control mechanisms. Internet Engineering Task Force (IETF). DOI: 10.17487/RFC4689

[15] DAHMOUNI, H., GIRARD, A., OUZINEB, M., et al. The impact of jitter on traffic flow optimization in communication networks. IEEE Transactions on Network and Service Management, 2012, vol. 9, no. 3, p. 279-292. DOI: 10.1109/TNSM.2012.051712.110148

[16] GELEJI, G., PERROS, H. Jitter analysis of an MMPP-2 tagged stream in the presence of an MMPP-2 background stream. Applied Mathematical Modelling, 2014, vol. 38, no. 14, p. 3380-3400. DOI: $10.1016 /$ j.apm.2013.11.055

[17] DBIRA, H., GIRARD, A., SANSO, B. Calculation of packet jitter for non-Poisson traffic. Annals of Telecommunications, 2016, vol. 71, no. 5-6, p. 223-237. DOI: $10.1007 /$ s12243-016-0492-0

[18] BRUN, O., BOCKSTAL, C., GARCIA, J. M. Analytic approximation of the jitter incurred by CBR traffics in IP networks. Telecommunication Systems, 2006, vol. 33, no. 1-3, p. 23-45. DOI: $10.1007 / \mathrm{s} 11235-006-9005-1$

[19] DONG, W., CHI, X. F., LIU, Z. C., et al. Queuing theory based analysis for packet jitter of mixed services. The Journal of China Universities of Posts and Telecommunications, 2014, vol. 21, no. 3, p. 71-76. DOI: 10.1016/S1005-8885(14)60303-4

[20] HAMMAD, K., MOUBAYED, A., SHAMI, A., et al. Analytical approximation of packet delay jitter in simple queues. IEEE Wireless Communications Letters, 2016, vol. 5, no. 6, p. 564-567. DOI: 10.1109/LWC.2016.2601609
[21] NA, F., CHI, X. F., DONG, W., et al. Jitter analysis of real-time services in IEEE 802.15.4 WSNs and wired IP concatenated networks. The Journal of China Universities of Posts and Telecommunications, 2016, vol. 23, no. 4, p. 1-8. DOI: $10.1016 / \mathrm{S} 1005-8885(16) 60039-0$

[22] ZHENG, Y., LU, K. J., WU, D. P., et al. Performance analysis of IEEE 802.11 DCF in imperfect channels. IEEE Transactions on Vehicular Technology, 2006, vol. 55, no. 5, p. 1648-1656. DOI: 10.1109/TVT.2006.878606

\section{About the Authors ...}

Xuefen CHI received the B.Eng. degree in Applied Physics from the Beijing University of Posts and Telecommunications, Beijing, China, in 1984, and the M.S. and Ph.D. degrees from the Changchun Institute of Optics, Fine Mechanics and Physics, Chinese Academy of Sciences, Changchun, China, in 1990 and 2003, respectively. She was a Visiting Scholar with the Department of Computer Science, Loughborough University, U.K., in 2007, and the School of Electronics and Computer Science, University of Southampton, Southampton, U.K., in 2015. She is currently a Professor with the Department of Communications Engineering, Jilin University, China. Her research interests include machine-type communications, indoor visible light communications, random access algorithms, delay-QoS guarantees, and network modelling theory and its applications.

Minghuan ZHANG was born in Hebei Province, China in December 1992. She received her B.Eng in the Dept. of Communication Engineering from Jilin University in 2016. Her research interests include wireless communication and delay jitter analysis.

Linlin ZHAO received the B.Eng., M.S. and Ph.D. degrees from the Dept. of Communications Engineering, Jilin University, Changchun, China, in 2009, 2012 and 2017, respectively. Her research interests include the throughput optimal random access algorithms, resource allocation schemes, delay analysis, and optimization in random access-aided wireless networks. In 2017, she has received the Best PhD Thesis Award of Jilin University.

Lei QIAN received her B.Eng. degree in the Dept. of Communication Engineering, Jilin University in 2016. She is currently working toward the Ph.D. degree in Jilin University. Her research interests include visible light communications and delay analysis. 